\title{
Embedding Emotional Context in Recommender Systems
}

\author{
Gustavo González, Josep Lluís de la Rosa, Miquel Montaner, Sonia Delfin \\ University of Girona. Institute of Informatics and Applications. Agents Research Lab. \\ Campus Montilivi. Building P4. E-17071, Girona, Spain. \\ \{gustavog; peplluis; montaner; slizzeth\}@eia.udg.es
}

\begin{abstract}
Emotions are crucial for user's decision making in recommendation processes. We first introduce Ambient Recommender Systems, which arise from the analysis of new trends on the exploitation of the emotional context in the next generation of recommender systems. We then explain some results of these new trends in real-world applications through the Smart Prediction Assistant (SPA) platform in an Intelligent Learning Guide with more than three million users. While most approaches to recommending have focused on algorithm performance, SPA makes recommendations to users on the basis of emotional information acquired in an incremental way. This article provides a cross-disciplinary perspective to achieve this goal in such recommender systems through a SPA platform. The methodology applied in $S P A$ is the result of a bunch of technology transfer projects for large real-world recommender systems.
\end{abstract}

\section{Introduction}

Over the last decade, research on recommender systems has focused on performance of algorithms for recommendations and improved ways of building user models to map user preferences, interests and behaviors into suggesting suitable products or services [2]. At the same time, several recommendation approaches and techniques have been developed in a variety of domain-specific applications [1]. Whereas many techniques have been proposed in user modeling, little attention has been paid on analyzing the emotional information involved in this modeling process.

In the real world, user's objective requirements can be forgotten and forsaken when his/her emotional needs are satisfied. The users always transmit their decisions together with emotions. For this reason, most consumer-centered companies are interested in detecting existing links between users' actions and the emotions produced in them by the companies' services, products and brands to automatically personalize and analyze user retention rates, loyalty rates, churn rates, cross-selling among other variables. Moreover, variables such as surprise, joy, disgust, empathy, feeling and, in general, users' emotional responses towards company products and services cannot be explicitly detected with current approaches. So, the questions that we try to answer in this article are: How to improve Recommender Systems and make them more pleasant to the user through the automatic perception of his/her emotional context? How can we build a more effective and satisfactory interaction in recommender systems? Can we automatically manage user's emotional awareness in recommender systems?

In order to answer these questions first, user models should be built with learning capabilities from user's emotional awareness in recommender systems. So, traditional approaches should require enriched support of future emerging information society technologies such as Ambient Intelligence and Pervasive Computing. Because of distributed, ubiquitous and open nature of this kind of future emerging technologies is needed the development of Smart Adaptive Systems [4] as a crucial component for modeling users with intelligent techniques that converge faster and in a more appropriate way than standard adaptive systems in the next generation of recommender systems [3]. Second, such recommender systems should learn from the user's interactions everywhere and anytime in non-intrusive way. Indeed, the complexity of modeling a user in real contexts is not a simple task, given that the context is a multidimensional parameter that includes time, location and subjectivity in perceptions and emotions [9].

With the vision of Ambient Intelligence (AmI) humans are the centre of new applications to discover, among others, emotional needs when users interact with ubiquitous recommender systems in everyday life [11]. An Ambient Recommender System proactively operates as a ubiquitous intelligent adviser on behalf of 
users in an everyday context; it is sensitive, adaptive and responsive to their needs, habits and emotions in real time while they reduce their information overload.

The article is structured as follows: In Section 2, we describe related work and new trends on the exploitation of the emotional context with a crossdisciplinary perspective to build user models in Ambient Recommender Systems. In section 3, we explain our methodology to manage emotions in recommender systems. Then we describe SPA (Smart Prediction Assistant) a customer intelligence platform that embeds the user's emotional intelligence in recommender systems. In Section 5, we test our approach in a large e-commerce Intelligent Learning Guide recommender system and we analyze some successful results. In Section 6, we facilitate a discussion about lessons learned with our deployment. Finally, we provide some conclusions and future work..

\section{Emotional Context in Recommender Systems}

It is well known that emotions play an essential role in user's decision making [10]. In particular, the user's emotional factor influences the rational thinking when a user receives any recommendation. The emotional factor is defined as the relevance that each user gives to differential values of items (i.e., events, services, products), which are demonstrated in the user's decision-making process by means of his or her actions.

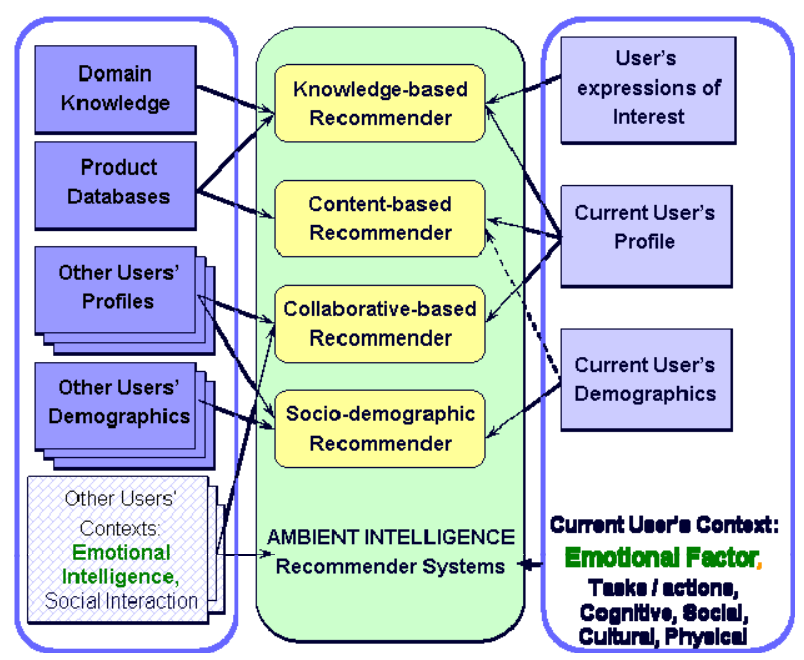

Fig. 1. Towards the next generation of Ambient Recommender Systems: an extended approach from R. Burke

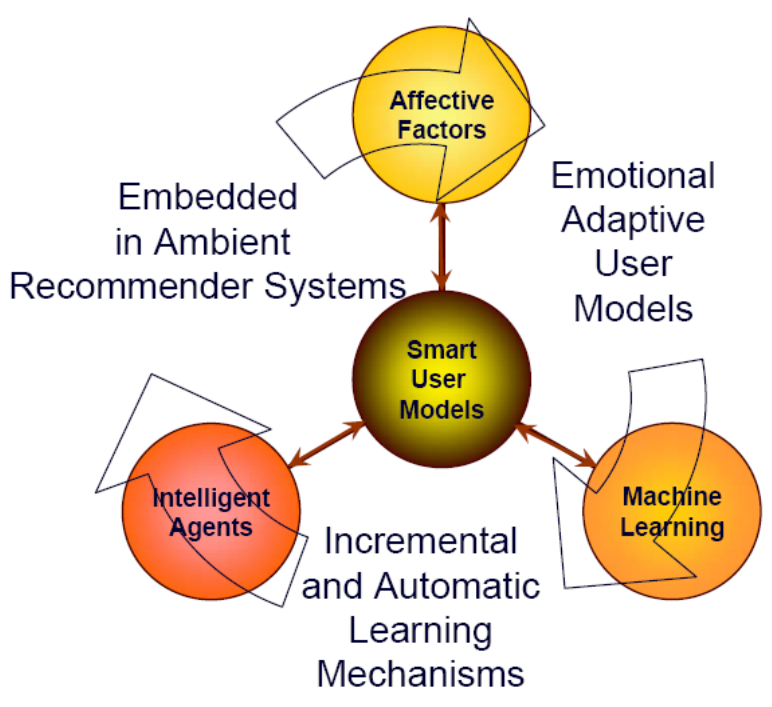

Fig. 2. Cross-disciplinary approach for embedding affective factors in recommender systems.

We extend correspondingly and tackle some challenges of the approach of Burke [2] surrounding knowledge sources and user profiles by users' context, in particular, the user's emotional context. As we show in the Fig. 1, this kind of Ambient Recommender Systems should represent in a holistic way several relevant properties (e.g., cognitive context, task context, social context, emotional context, cultural context, physical context and location context among others variables) according to the user's circumstances, using existing and new techniques for recommendations. For instance, the daily life of nomadic users of recommender systems in the home environment, workplace, car, leisure time and tourism, among other scenarios, can be simplified and enriched by satisfying their interests, preferences and understanding their emotional context.

In order to accomplish the above mentioned objectives we have developed a synergistic approach which combines a model of the user's emotional information with machine learning and intelligent agents (see Fig. 2) in the so-called Smart User Models (SUMs) [5]. They act like unobtrusive intelligent user interfaces to acquire, maintain and update the user's emotional information through an incremental learning process in everyday life.

In particular, SUMs can provide to Ambient Recommender Systems the capability to manage the 
user's emotional context, acquiring his or her emotional intelligence. This capability is becoming increasingly important for modeling humans holistically (i.e. taking into account all spheres of their everyday life, with their skills and weakness to interact with a plethora of recommender systems in multiple domains) in engaging applications to improve their interaction in a more effective and satisfactory way. Consequently, the following properties have been taken into account in our methodology to achieve tailored personalization:

- Smartness provided by users' affective factors and context-awareness by integrating emotional capabilities.

- Human-centered interaction improved by agents that act on behalf of users in flexible ways (i.e. in proactive, reactive and social ways). Through a multiagent architecture SUM is also able to resolve the proactive and autonomous pre-processing of raw data acquired from user's multiple interactions in several application domains and in a multi-modal way.

- Continuous modeling through machine learning techniques in open environments. SUM learns in an incremental way from users' continuous interactions in complex situations in which the emotional context is relevant to suggestions made by the recommender systems.

Some other approaches in recommender systems consider contextual information associated with the ambient intelligence scenarios. For instance, relevant research work is being carried out on users' emotional factors in recommender systems [7] to better understand users in complex situations. At the same time, challenging research initiatives for defining standards in emotion-oriented computing are becoming increasingly relevant [12].

Currently, there are few commercial recommender systems which consider these sensibilities for creating user models. Most systems use statistical techniques. Some others use data mining techniques mixed with relational marketing concepts to create behavior patterns of users and consumers and then classify them according to rules. However, none of these recommender systems consider the emotional context as fundamental component of analysis to create truly personalized and individualized user models according to preferences and interests. Most importantly, they do not have capabilities for smart behavior, that is, they should be able to learn in an incremental way and produce effective recommendations in a wide variety of complex circumstances.

\section{Automatic Learning of Emotional Context through User's Emotional Intelligence}

According to Salovey and his colleagues emotional intelligence is "a type of social intelligence that involves the ability to monitor one's own and others' emotions, to discriminate among them, and to use the information to guide one's thinking and actions" [8]. Emotional Intelligence can be measured, ranging from feelings of boredom to feelings of happiness and euphoria, from hostility to fondness.

\section{Branch Name Brief Description of Skills Involved}

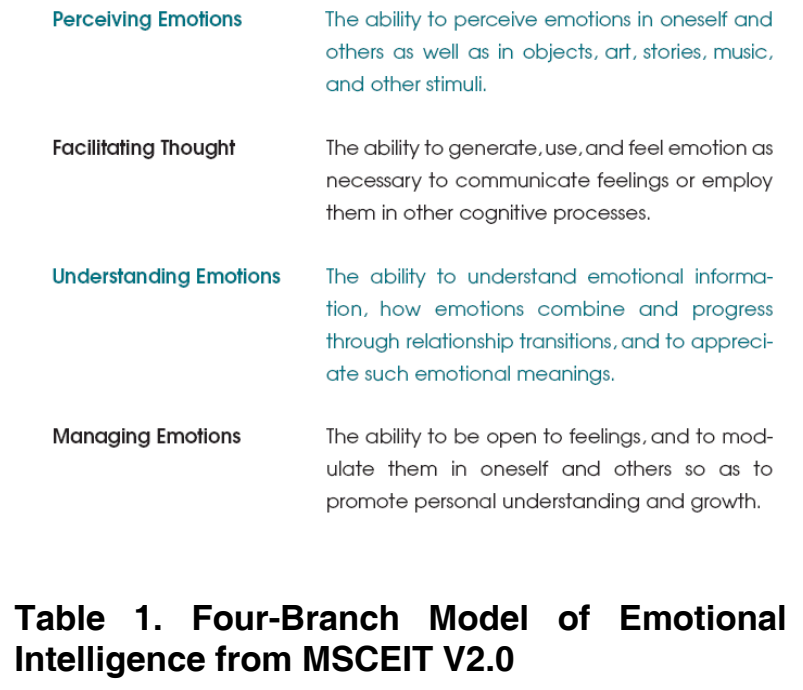

Managing Emotions

The ability to be open to feelings, and to modulate them in oneself and others so as to promote personal understanding and growth.

\section{Table 1. Four-Branch Model of Emotional Intelligence from MSCEIT V2.0}

We have taken into account these parameters for developing socially intelligent recommender systems as a critical step towards enabling them to be more intuitive with the user since there is an obvious link between personality traits and user preferences - both being indications of default tendencies in behavior. Our methodology provides a high added value to existing approaches in recommender systems through the users' emotional information, so we highlight the relevant stages to acquire, manage and update the emotional information about users:

1. Initialization stage: this stage consists of the acquisition of users' emotional features based on a gradual and noninvasive emotional intelligence test (i.e Gradual EIT). Particularly, in this work we apply the so-called the Four-Branch Model of Emotional Intelligence (see Table 1) which can be measured through the Mayer-Salovey-Caruso Emotional Intelligence Test (MSCEIT V2.0) [8]. Particularly we emphasize that each emotional state can be labeled 


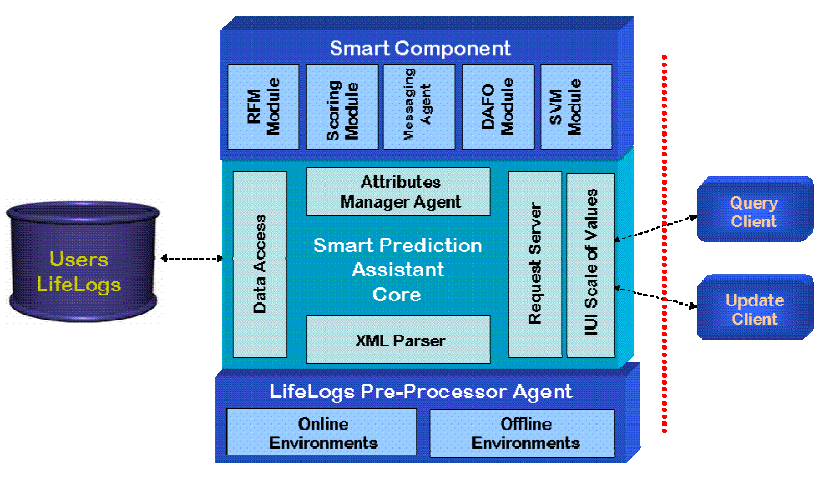

Fig. 3. Architecture of Smart Prediction Assistant.

with a valence. In the context of emotional information a valence is the degree of attraction or aversion that a person feels toward a specific object or event

2. Advice stage: this stage consists of providing emotional information to recommender systems to improve recommendations made to the user. It is based on activation or inhibition of excitatory attributes from each domain of interaction according to the emotional information.

3. Update stage: this stage keeps the SUM informed of user changes according to recent interactions based on reward and punish mechanisms.

For the sake of extension, we encourage the reader to consult details on the formal definition of SUM and the methodologies developed in [5].

\section{Smart Prediction Assistant: Embedding Emotions in Real-World Recommender Systems}

Smart Prediction Assistant (SPA) is an innovative customer intelligence platform based on technology transfer results from several $R \& D$ projects in which results with high impact on the e-commerce domain have been obtained. The SPA customer intelligence platform is an advance in the evolution of HabitatPro $^{\text {TM }}$ V2.5, which was a supervised platform to batchprocess user profiles. SPA is now a semi-supervised platform that automates several time-consuming manual processes (i.e. the tasks of data selection, data gathering and data pre-processing) in both off-line and on-line environments to build dynamic and emotional user models for recommender systems. SPA improves the existing platform, embedding powerful incremental learning mechanisms to create highly accurate emotional intelligence-based models. To achieve this objective SPA exploits heterogeneous, multi- dimensional and massive databases to extract, preprocess and deliver distilled user LifeLogs.

The user's LifeLog is a complex set of all the raw data about the user generated by the interaction with multiple applications in several domains in a multimodal way (i.e. socio-demographic data, web usage data, transactional data, explicit ratings, attribute databases, physiological signals, etc). The user's Lifelog is captured and extracted from user's interaction with several applications in the real world. Lifelog is gathered by the continuous storage of raw information streams which are provided by surroundings knowledge sources in ubiquitous recommenders systems.

With SPA the scalability has been improved from hundreds of thousands of users to millions of users. Thus, SPA can suggest in an automatic way individualized messages for each user. SPA is also able to create in real-time and in an automatic way individualized Human Values Scales for each user according to his/her dominant attributes.

The following components have been developed in SPA (see Fig.3):

1. LifeLogs Pre-processor Agent: This agent replicates itself in pro-active way depending of user's interaction with several applications. Its function is to pre-process raw data in on-line and off-line environments.

2. Smart Component: This component implements advanced algorithms and methods for incremental learning in order to accurately predict user behavior. It has graphics tools to monitor and manage scorings, classifications, rankings of attributes, items and users, user propensity and others capabilities.

3. Attributes Manager Agent: This agent is able to create, extract, select, and fuse attributes in order to evaluate similar attributes for multiple domains of interaction and also to contrast them in an automatic way. This agent automatically detects the level of sensibility of each user for each of his/her dominant attributes by automatically assigning weights (relevancies).

4. Messaging Agent: This agent is able to automatically generate emotional arguments from users' dominant attributes by using messages in each application domain for each product. This agent acts on behalf of marketing retailers to define individualized communication styles for each user.

5. Intelligent User Interface: It is an add-on component to manage an individualized and personalized Human Values Scale of each user in his/her life cycles. It embeds an intelligent feedback mechanism that enables: 


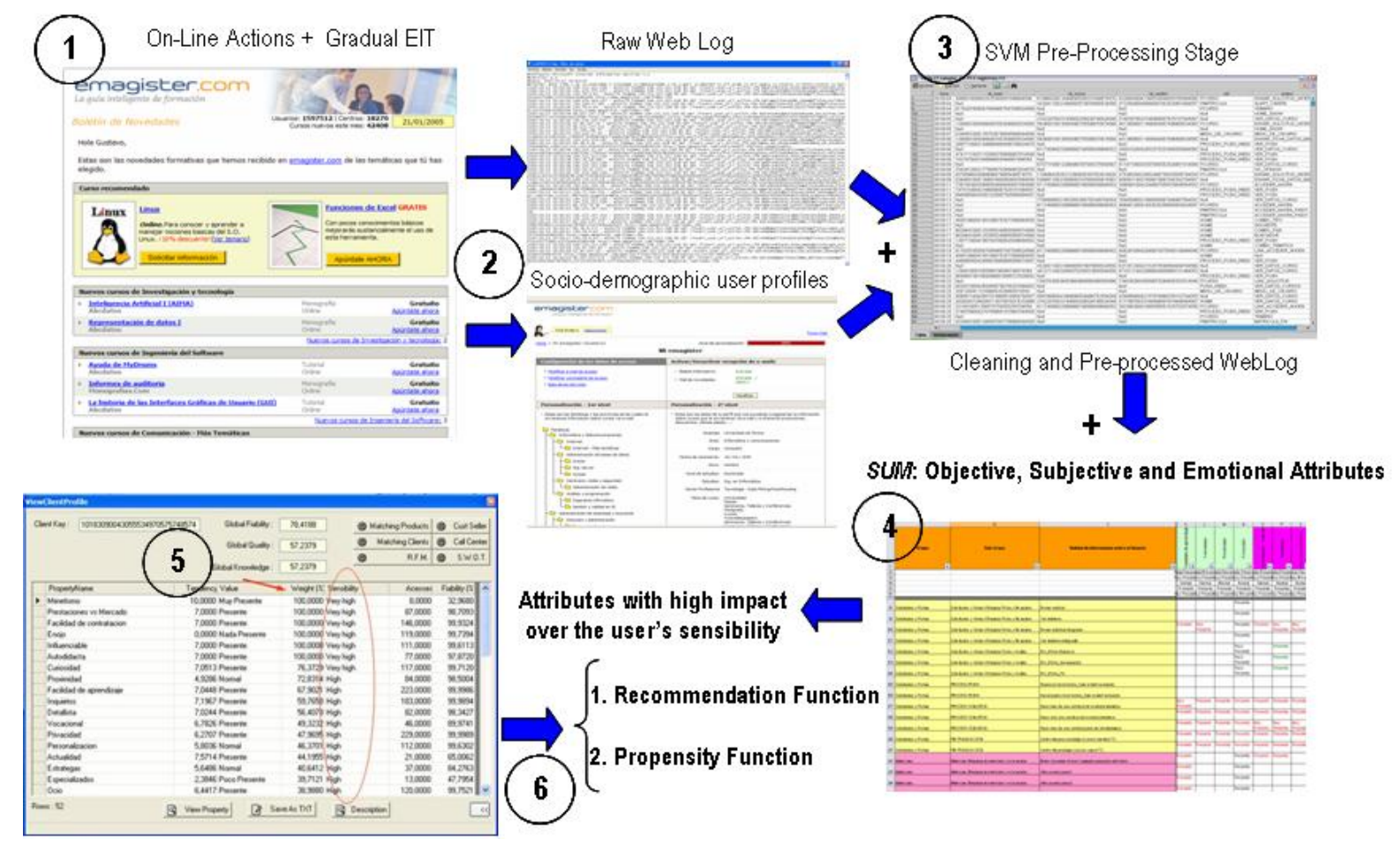

Fig. 4. Iterative automatic process to discover, manage and update emotional attributes in SPA.

a. The analysis of diverse values from the individualized scale of each user in real time.

b. The definition of the coherence function between a user's actions and his/her implicit and explicit preferences in a recommender system.

For reason of clarity, in this article we only describe our deployment using the first four components of SPA. For details about methodology implemented in the fifth component of SPA we encourage the reader to consult details in [6].

\section{A Business Case: The Intelligent Learning Guide Recommender System}

We have deployed SPA in a large e-Commerce Intelligent Learning Guide with more than three million users, namely emagister.com. Usually, in this kind of recommender system, the suggestions are delivered through a combination of the user's explicit preferences and user feedback is acquired via clickstream analysis (i.e., implicit feedback) and/or user rating of the perceived quality of recommended items (i.e., explicit feedback), generating raw data to be analyzed. The user's decision making has been enriched and supported by measuring his or her sensibility to specific attributes in specific domains of training. In particular, not only are users advised in selecting the more relevant course to satisfy their preferences and interests, but they also intensify their satisfaction by discovering their sensibility to particular attributes that allow better communication via newsletters. In this scenario, SPA take advantage of the SUMs capturing both objective features of users and information related to their subjectivity in ecommerce applications.

\subsection{Data Description}

In this case, SUM gathers 75 objective, subjective and emotional attributes of 3,162,069 registered users till 14th March of 2006. The set of possible on-line user's actions on the web of emagister.com was 984 . Data has been extracted from socio-demographic databases (i.e. user profiles with objective attributes) and WebLogs of user's implicit navigation habits (i.e subjective and emotional attributes discovered according to the users' answers given in the Gradual EIT). Specifically, we have ten suitable emotional attributes with different kind of valence for this 
business case: enthusiastic, motivated, empathic, hopeful, lively, stimulated, impatient, frightened, shy and apathetic. WebLogs are close to $50 \mathrm{~Gb}$ /month.

\subsection{Process Description}

First, a marketing strategy was designed whereby emotional attributes and their values are collected using the Gradual EIT implemented for each user through push and newsletters communications. When users answer questions (only one question every time that push or newsletters are received) related to their common day to day situations (opinions, tastes, pictures, etc), their impacted emotional attributes related with the questions are gradually activated [3]. Finally, in order to maintain their emotional attributes and values updated each time that users open and surf the recommendation sent in Push or newsletters communications about training courses, the reward mechanism works to reinforce the related attributes and values (see Fig. 4). Thus, users' emotional responses are discovered from their interactions in a dynamic and non-intrusive way in order to improve recommendations regarding training courses. It is important to note that in many occasions users do not answers questions which produce lack of relevance feedback from the user side and the effect known as the sparsity problem in data. To reduce the dimensionality of the matrix generated we use Support Vector Machines (SVM). Then SVMs are used to classify and to predict users' behaviors from attributes which have a high impact on their emotional responses. Furthermore, SVMs have been used as a learning component in ranking users to assess their propensity to accept a recommended item.

\subsection{Persuading Users through their Sensibilities}

Outstanding salesmen use a different sales talk depending on the customer. Salesmen communicate a different message depending on customers' sensibilities, since if they catch their attention the sale is easier. What the Messaging Agent tries to do is to simulate this salesmen behavior.

Therefore, we generate a personalized message to each user following the next steps based on the users' sensibilities:

1. Select the product attributes to be used for the sales talk: depending on the features of the training course to sale, we select from the list of attributes the ones that can be used to sell the course.
2. Generate a message (sales talk) for each product attribute: this generation is carried out once and then is saved in a database of messages.

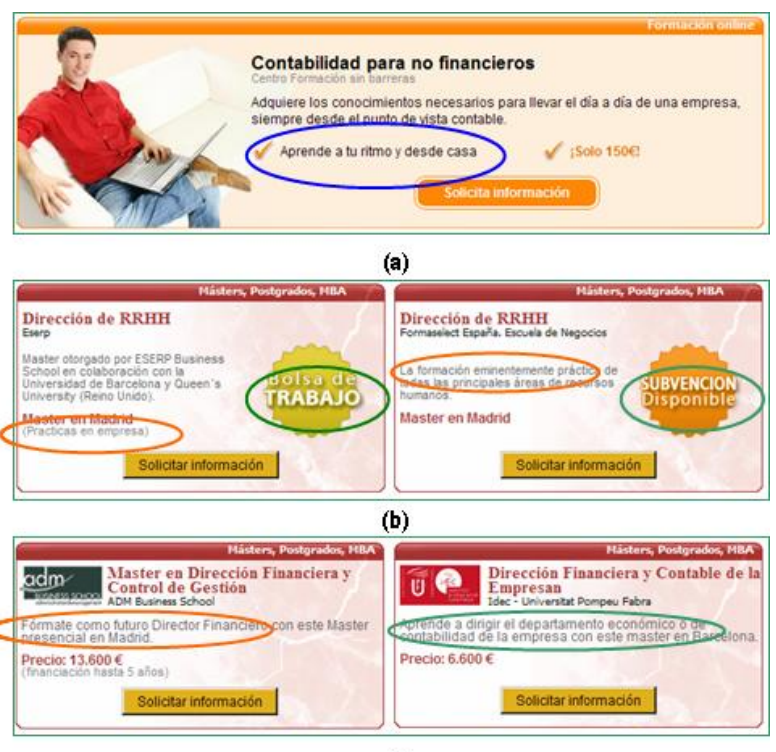

(c)

Fig. 5. Samples of individualized messages for each user according his/her dominant sensibilities: (a) case with only one attribute that impact over user's sensibility. (b) case with several attributes ordered by priority and (c) case with several attributes assigning message with most sensibility.

3. Assign a message to each user depending on his/her sensibilities: that is, the attributes of his/her user

model that exceed a sensibility threshold. Then, we match these sensibilities with the attributes selected for the training course. In this moment, we can get three different cases:

a. The user does not have any sensibilities for product attributes: a standard message is assigned to this user.

b. The user has one of the product attributes as sensibilities: the message of this product attribute is assigned to the user automatically.

c. The user has more than one of the product attributes as sensibilities: here we have two options:

i. Order product attributes by priority and assign to the user the message of the attribute with most priority.

ii. Assign to the user the message of the attribute that the user has most sensibility. 


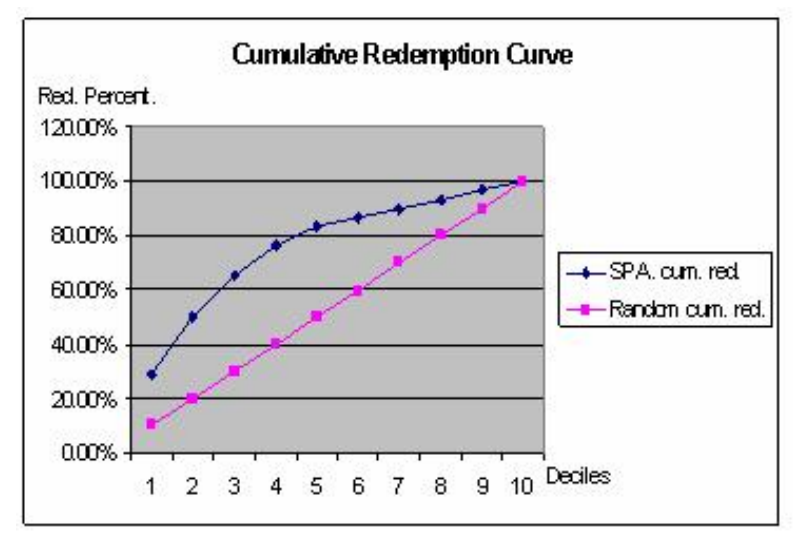

(a)

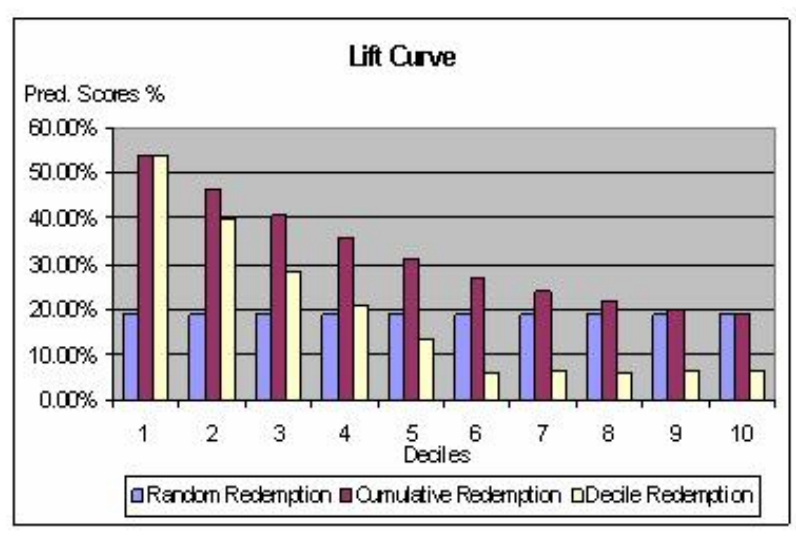

(b)

\section{Fig. 6. Cumulative redemption curve (a) and Predictive Scores (b) for the increase in transactions of $1,340,432$ users of emagister.com}

Fig. 5 shows different kind of messages sent by the Messaging Agent for each user. In Fig. 5(a) the user has very much sensibility for the emotional attribute enthusiastic (i.e. the case 3.b.). In Fig. 5(b) the user has sensibility for four emotional attributes which are ordered by priority: lively, stimulated, shy and frightened (i.e. the case 3.c.i.). Finally, in Fig. 5(c) the user has sensibility for two emotional attributes: motivated and hopeful, however in this case the Messaging Agent assigned to the user the message with the attribute hopeful, which impact most the user's sensibility (i.e. the case 3.c.ii.).

\subsection{Results}

We have tested SPA with eight Push and two newsletters campaigns. The target was 1,340,432 users in each campaign chosen in random way. Our objective was to predict the users' propensity to do transactions (i.e. actions such as click streams, information requirement about training courses, enrollments, opinions, etc.) in Push and newsletter communications. Thus, SPA delivered more empathic recommendations through two well differenced functions:

1. The recommendation function: to send in an individualized manner the action with most probabilities of execution by the user.

2. The selection function: to choose the user with greater propensity to follow a course in the recommender system.
Both functions are based on attributes which have high impacts on users' emotional sensibility to predict their behaviors.

Fig. 6 shows successful results obtained with our predictive models tested with a target group of users in the overall set of ten campaigns. Particularly, Fig. 6(a) shows that with the $40 \%$ of commercial action (i.e. the effort to send Push and newsletters), SPA achieves more than $76 \%$ of useful impacts.

So, we have improved the redemption of Push and newsletters campaigns in a $90 \%$. On the other hand, Fig. 6(b) shows the predictive scores of the total set of ten Push and newsletters campaigns. So, SPA achieves an average performance of $21 \%$, it means 282,938 useful impacts.

\section{Lessons Learned}

In this work a domain with high level of collaboration was used to test SPA. We are aware that SPA is more appropriate for companies that can acquire user's implicit feedback through a rich interaction through highly dynamic environments. For instance, networked games and leisure (e.g. music and tourism recommender systems) are potential application domains for SPA. The question however remains open for domains where the user is not willing to collaborate with the system. In this sense, acquiring relevant information to keep user's preferences up-todate is crucial in order to close the cycle of recommendations and to obtain successfully results using SPA. From our experience, we have learned that SPA is particularly suitable for domains where users 
perceive that the value of the information delivered from company/system side is higher than the value of the information delivered from their side. Instances of this kind of systems are social software systems which organize contextual information about user activities and tasks in all spheres of information (i.e. at home, at work, travels, relationships and shared interests, among others).

\section{Conclusions}

SPA is a smart adaptive system based on the integration of intelligent agents with adaptive and incremental learning capabilities to embed the user's emotional intelligence in recommender systems. It was developed through a multi-disciplinary approach inspired in future emerging recommender systems in Ambient Intelligence scenarios.

Along these lines, we have suggested a shift in the way that research community on recommender systems could direct efforts to build more intuitive and sensitive systems.

The incremental advance in SPA is its capability to determine high-accurate predictions not only based in objective attributes but also on subjective and emotional attributes acquired from user interactions in recommendation processes. This product is the first software tool created in its category that embeds users' emotional information to enrich recommendations in every day life.

SPA also reports several business benefits for enterprises interested in discovering users' emotional sensibilities associated to their services and products.

SPA has high performance pre-processing proactively LifeLogs of millions of customers. SPA also includes smart behavior in its user models which allow reduced costs of maintenance by experts. Finally, SPA is able to generate individualized messages for each customer according to his/her life cycles during the purchase processes.

In further work, we will improve SPA with unobtrusive methods of acquisition of user's relevant feedback in real time. We are sensing physiological and contextual parameters of firefighters in Paris brigades through wearable computing in the wearIT@work project to provide recommendations to their commander who is advised by an Ambient Recommender System in an emergency (such as fire, earthquake, tsunami or missing people) based on the firefighter's emotional information. These experiments are being carried out with the SPA platform mapping physiological signals to user's emotional context. The objective of the team commander is to receive advice from the system about firefighter's current emotional state and its implications in the rescue operation so he can better assess the operational fitness of his colleague in particular situations.

\section{Acknowledgments}

This research was supported by the European Project (ONE) - Open Negotiation Environment, $\mathrm{N}^{\mathrm{o}}$ 34744, FP6-2005-IST-5, ICT for Networked Businesses.

\section{References}

[1] Adomavicius, G. and Tuzhilin A. 2005. Towards the Next Generation of Recommender Systems: A Survey of the State-of-the-Art and Possible Extensions. IEEE Transactions on Knowledge and Data Engineering 17(6): 734,749.

[2] Burke, R. 2001. Hybrid Recommender Systems: Survey and Experiments, User Modeling and User-Adapted Interaction 12(4): 331-370.

[3] Doom, C. 2001. Get Smart: How Intelligent Technology Will Enhance Our World. CSC's Leading Edge Forum Report. Available on-line at: http://www.csc.com/aboutus/lef/mds67_off/uploads/getsmart -final.pdf

[4] Gabrys. B., 2005. Do Smart Adaptive Systems Exist? Gabrys, Bogdan; Leiviskä, Kauko; Strackeljan, Jens (Eds.). Springer-Verlag. ISBN: 978-3-540-24077-8.

[5] González, G., López, B. and de la Rosa J. LL. 2005. A Multi-agent Smart User Model for Cross-domain Recommender Systems. In Proceedings of Beyond Personalization 2005: The Next Stage of Recommender Systems Research, held in conjunction with IUI'05. San Diego, California, USA.

[6] Guzmán, J., et al. 2005. Modelling the Human Values Scale in Recommender Systems: A First Approach. in Artificial Intelligence Research and Development: 405-412. Radeva, J., et al. Eds. IOS Press. 131. The Netherlands.

[7] Masthoff, J. 2005. The Pursuit of Satisfaction: Affective State in Group Recommender Systems. In Proceedings of User Modeling 2005: 297-306. Brna, P., Ardissono, L. and Mitrovic, A. Eds. LNAI. Springer Verlag, Berlin.

[8] Mayer, J.D., et al.. 2003. Measuring Emotional Intelligence with the MSCEIT V2.0. Emotion: 3(1). 97-105.

[9] Minsky, M. 2006. The Emotion Machine: Commonsense Thinking, Artificial Intelligence, and the Future of the Human Mind. ch 1, 2, 7. New York: Simon \& Schuster.

[10] Picard, R. W. et al. 2004. Affective Learning - a manifesto. BT Technology Journal 22(4): 253-269.

[11] Remagnino, P. and Foresti, G.L. 2005. Ambient Intelligence: A New Multidisciplinary Paradigm. IEEE Transactions on Systems, Man and Cybernetics - Part A: Systems and Humans 35(1): 1-6.

[12] W3C Emotion Incubator Group. Available: http://www.w3.org/2005/Incubator/emotion/ 